\title{
Invasive Gehölzinsekten: Bedrohung für den Schweizer Wald?
}

Beat Wermelinger

\author{
Eidgenössische Forschungsanstalt für Wald, Schnee und Landschaft $(\mathrm{CH})^{*}$
}

\section{Invasive tree insects: a menace to the Swiss forests?}

\begin{abstract}
The rate of introduction of non-native insects and other arthropods to Europe has been exponentially increasing in recent decades, amounting to 20 species per year in the past ten years. By 2008, a total of 1590 species had been introduced since the discovery of America. The main causes are the intensification and globalization of international trade. The most important pathways are trade in ornamental plants and import of goods in infested wooden crates. Most often new species first establish in residential areas, where the normally mild climate favors the survival of exotic species. Three species recently introduced from East Asia to Switzerland are particularly relevant to forests: the box tree moth (Cydalima perspectalis) has infested box tree stands near Basel, the chestnut gall wasp (Dryocosmus kuriphilus) now covers almost all of southern Switzerland, with single infestations also on the Swiss Central Plateau, and two infestation spots of the Asian longhorned beetle (Anoplophora glabripennis) have been identified. Potential future species of invasive insects are discussed. The largescale introduction of foreign tree species in forests needs to be critically examined, as they may be hosts of current and future non-native insects.
\end{abstract}

Keywords: non-native insects, alien insects, international trade, Anoplophora, Dryocosmus, Cydalima doi: $10.3188 /$ szf.2014.0166

*Zürcherstrasse 111, CH-8903 Birmensdorf, E-Mail beat.wermelinger@wsl.ch

$\mathrm{I}$ $\mathrm{n}$ den letzten Jahrzehnten machten sich weltweit immer mehr gebietsfremde Arten bemerkbar, die als invasiv bezeichnet werden müssen und somit ein ökonomisches oder ökologisches Risiko darstellen. Die Einschleppungen von Arthropoden (Gliederfüsser; davon $87 \%$ Insekten) nach Europa haben in den letzten zwei Jahrhunderten exponentiell zugenommen (Abbildung 1): Waren es vor hundert Jahren rund sieben neu etablierte Arten pro Jahr, sind es heute fast 20 Arten. Seit der Entdeckung Amerikas, die als Beginn des weltweiten Warenaustausches betrachtet wird, etablierten sich bis 2008 insgesamt 1590 fremde Arthropoden in Europa (Tabelle 1), vor allem Käfer, Blatt- und Schildläuse sowie Hautflügler. Dies entspricht etwa 1 bis $2 \%$ der in Europa heimischen Arten. Knapp ein Drittel der herbivoren (pflanzenfressenden oder -saugenden) Arten stammt aus Asien und ein gutes Viertel aus Nordamerika (Roques 2010a). Etwa ein Drittel der eingeschleppten Insekten lebt an Gehölzpflanzen (Tabelle 1).

Abbildung 2 zeigt die zeitliche Entwicklung der Einschleppung von gebietsfremden Insekten in die Schweiz, basierend auf der Liste von Kenis (2006) und aktualisiert mit eigenen Daten. Dabei wurden Insekten mit mediterraner Herkunft weggelassen (eventuell klimabedingte Einwanderung) und nur diejenigen Arten berücksichtigt, die lebende Gehölze befallen und von denen das Jahr der Einschleppung in die Schweiz eruiert werden konnte. Die kumulative Anzahl eingeschleppter Arten zeigt, dass die Einschleppungen im Wesentlichen in den 1930er-Jahren begannen. Die Einschleppungsrate pro Jahr blieb bis in die 1970er-Jahre ungefähr konstant und nahm danach bis heute zu. Die wichtigsten Gründe für die Verschleppung von Arten über geografische Barrieren hinweg sind der in den letzten Jahrzehnten massiv zunehmende internationale Warenhandel und die fast grenzenlose Mobilität des Menschen. So korrelieren die Einschleppungsraten von invasiven Organismen bestens mit der Menge der importierten Güter (Westphal et al 2008). Dies gilt auch für die Schweiz, wo das Importvolumen aller importierten Güter seit Mitte des letzten Jahrhunderts kontinuierlich zunahm, mit zwei Zwischenmaxima um 1980 und 1990 (Abbildung 2). 


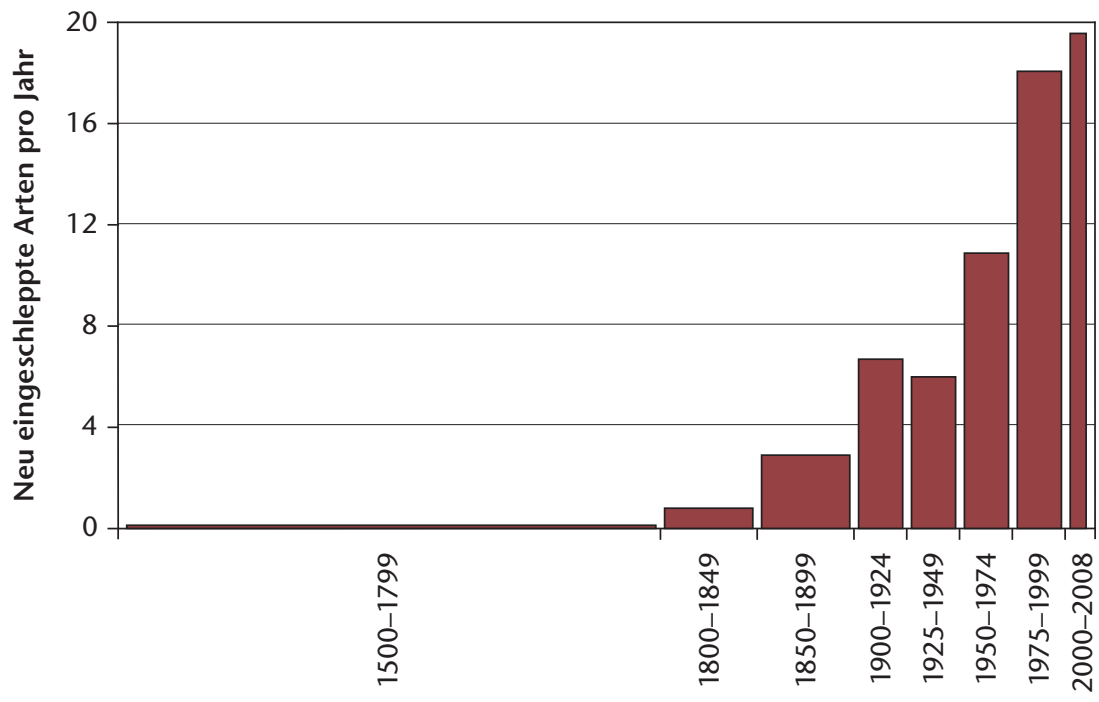

Abb 1 Jährliche Einschleppungen von neuen Arthropodenarten (v.a. Insekten und Milben) nach Europa seit der Entdeckung Amerikas (verändert nach Roques 2010a).

\begin{tabular}{|l|c|c|l|}
\hline & Europa & Schweiz & Quelle \\
\hline $\begin{array}{c}\text { Arthropoden } \\
\text { Insekten }\end{array}$ & 1590 & & Roques 2010a \\
\hline $\begin{array}{c}\text { Gehölzinsekten } \\
\text { Käfer }\end{array}$ & 1390 & 311 & Roques 2010a, Kenis 2006 \\
\hline
\end{tabular}

Tab 1 Anzahl der in den letzten 500 Jahren (bis ca. 2004) nach Europa und in die Schweiz eingeschleppten Arthropoden (v.a. Insekten und Milben).

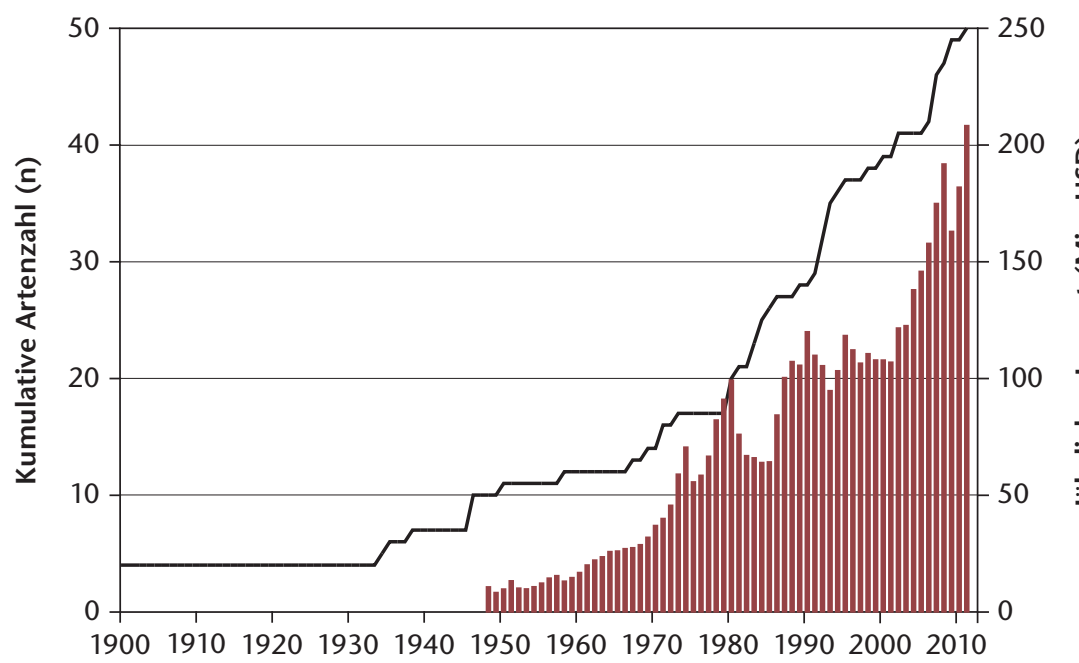

Abb 2 Kumulative Zahl der in die Schweiz eingeschleppten Gehölzinsekten (Kurve) und inflationsbereinigter Importwert der in die Schweiz eingeführten Handelsware (Säulen; Quelle: Datenbank WTO, http://stat.wto.org [25.10.2013]; inflationsbereinigt auf das Jahr 2011 mit dem US-Inflationsrechner, www.usinflationscalculator.com [28.10.2013]).

\section{Einschleppungswege und Verbreitung}

Der wichtigste Einschleppungsweg für an Gehölzpflanzen lebende Insekten ist der Import von Holzprodukten oder Zierpflanzen (Roques 2010b). In Grossbritannien ist der Zierpflanzenhandel für fast 90\% der Einschleppungen von herbivoren Insekten verantwortlich (Smith et al 2007). Larven in Holz und Wurzeln oder Eier sind schwer zu entdecken und werden deshalb bei der Kontrolle leicht übersehen. Der Zierpflanzenbereich umfasst Bäume und Sträucher für Privatgärten, Baumschulen, Grossabnehmer oder auch internationale Grossanlässe, für die grosse Bäume mit Wurzelballen eingeführt und gepflanzt werden. Ein weiterer wichtiger Einschleppungsweg ist das Verpackungsholz von beispielsweise Steinprodukten und Maschinen. Kisten, Lattenverschläge und Paletten können Larven von xylophagen (holzfressenden) Insekten beherbergen, die ihre Entwicklung am Bestimmungsort vollenden und dort ausschlüpfen.

Meistens treten fremde Gehölzinsekten zuerst in Siedlungsgebieten auf. Dies hat verschiedene Gründe: In Siedlungen werden die meisten importierten Zierbäume angepflanzt, es stehen häufig bereits weitere exotische Wirtspflanzen zur Verfügung, viele Stadtbäume haben eine reduzierte Widerstandskraft gegen Befall (Trockenheit, Schadstoffe, Sauerstoffmangel der Wurzeln, mechanische Schäden usw.), es herrscht ein milderes Klima als in der offenen Landschaft oder im Wald, und in Ballungsräume werden die meisten in Holz verpackten Waren geliefert. 46\% der bisher eingeschleppten Arten verweilten auf ihren ursprünglichen, exotischen Wirten und gingen nicht auf einheimische Bäume über (Roques 2010b). 15\% der Arten breiteten sich auch in Waldgebiete aus (Roques 2010b). Nach der Etablierung kann sich eine Art natürlich (oft vom Wind unterstützt) oder auch durch den Binnenhandel mit befallenem Pflanzenmaterial, durch Verschleppung mit Fahrzeugen und durch die menschliche Reisetätigkeit ausbreiten.

Einzelne invasive Insekten wurden auch absichtlich eingeführt. Der Asiatische Marienkäfer (Harmonia axyridis) zum Beispiel wurde zur biologischen Bekämpfung von Blattläusen in Gewächshäusern und Obstanlagen von Ostasien nach Europa importiert. Mittlerweile hat er sich verselbstständigt und ist in vielen Ökosystemen Europas die dominante Marienkäferart geworden. Viele einheimische Marienkäferarten wurden dadurch deutlich zurückgedrängt (Roy et al 2012).

Das Erscheinen neuer Organismen in einem Gebiet kann aber auch klimatische Ursachen haben. Der laufende Klimawandel schafft für viele temperaturlimitierte Arten günstige Bedingungen, was dazu führt, dass sie ihr Verbreitungsareal nach Norden und in die Höhe ausdehnen können. So hat sich der auch in der Schweiz heimische Pinienprozessionsspinner (Thaumetopoea pityocampa) in Frankreich stark nach Norden und in Spanien in höhere Lagen ausgebreitet, mit negativen Folgen für die neu betroffenen Föhrenbestände (Battisti et al 2005). Auch in der Schweiz gibt es verschiedene mediterrane Gehölzinsekten, die sich auf die Alpennordseite ausgebreitet haben. Beispiele dafür sind die Südliche Eichenschrecke (Meconema meridionale) oder die Malvenwanze (Oxycarenus lavaterae). 


\section{Einige wichtige invasive Arten in der Schweiz}

Etliche fremde Insektenarten haben sich schon seit einiger Zeit auf Bäumen und Sträuchern in der Schweiz etabliert und sind, grösstenteils völlig unauffällig, zu einem Bestandteil der hiesigen Fauna geworden (Liste siehe Kenis 2006). Für den Wald sind vor allem Käfer und Pflanzenläuse relevant. Unter den sechs eingeschleppten Borkenkäferarten hat vor allem der Schwarze Nutzholzborkenkäfer (Xylosandrus germanus, seit 1984 in der Schweiz) an gelagertem Holz eine gewisse Bedeutung erlangt. Bei den Läusen sind es die Weisstannentrieblaus (Dreyfusia nordmannianae, 19. Jahrhundert) und die Douglasienwolllaus (Gilletteella cooleyi, 1930er-Jahre). Auf Bäumen im urbanen Bereich spielen verschiedene Miniermotten an Robinie (Robinia pseudoacacia), Rosskastanie (Aesculus hippocastanum) und Platane (Platanus spp.) eine gewisse Rolle. Drei kürzlich in die Schweiz eingeschleppte Arten, welche auch im Wald grössere Bedeutung erhalten könnten oder schon erhalten haben, werden im Folgenden vorgestellt.

\section{Buchsbaumzünsler}

Dieser ostasiatische Nachtfalter wurde in der Schweiz erstmals 2007 im Raum Basel festgestellt und verbreitete sich durch den Verkauf befallener Buchspflanzen rasant über das ganze Land. Die Raupen des Buchsbaumzünslers (Cydalima perspectalis) fressen an Buchs (Buxus sempervirens) und können eine Pflanze in kürzester Zeit entlauben. Befallssymptome sind Spinnfäden, Kot und vergilbte, vertrocknete Blattreste (Abbildung 3). Bei hoher Raupendichte fressen die Tiere auch an der grünen Zweigrinde, was zum Absterben des befallenen Triebs führt. Bei wiederkehrendem Befall ist die Überlebensrate einer Pflanze trotz Neuaustrieb ohne Insektizideinsatz tief, weshalb der Buchs nicht mehr als anspruchslose Zierpflanze betrachtet werden kann.

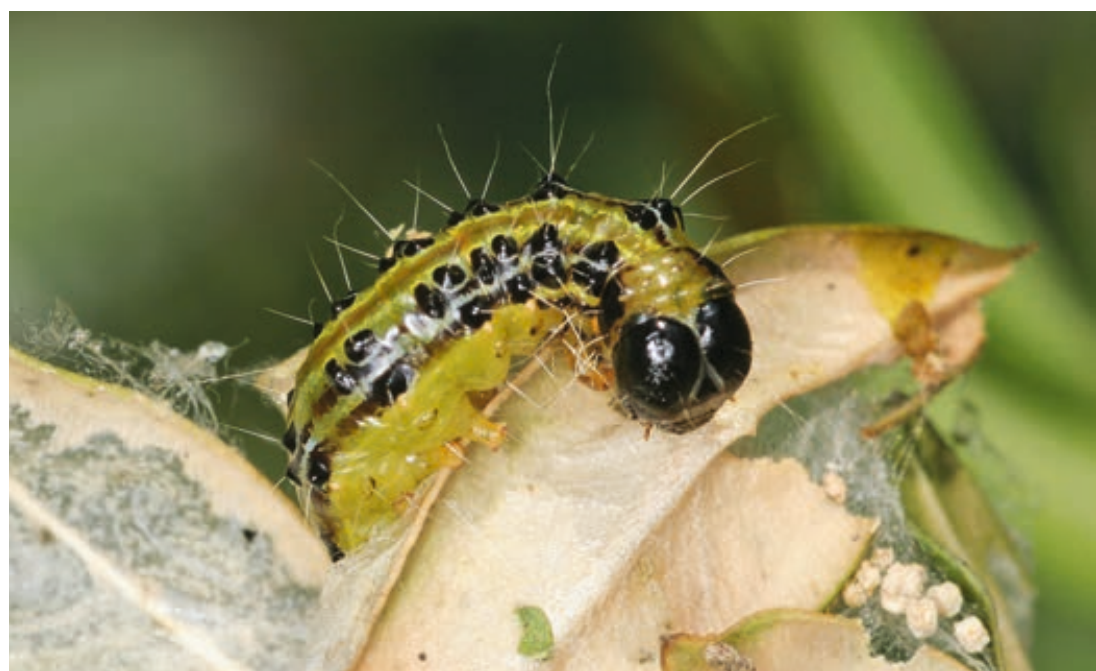

Abb 3 Raupe des Buchsbaumzünslers (Cydalima perspectalis) mit den typischen Frasssymptomen: angefressene, vergilbte Blätter mit Kot und Spinnfäden.
Der Buchsbaumzünsler befiel ab 2010 im Raum Basel auch natürliche Buchsbestände im Wald. Diese wurden völlig kahl gefressen und erholen sich seither nur schlecht (Meier et al 2013). In einem angrenzenden, deutschen Waldgebiet mit grossflächigem Buchsunterwuchs waren von 2010 bis 2012 alle Buchspflanzen zu mindestens 90\% entlaubt. 27\% erlitten Totalfrass und überlebten den Befall nicht (Kenis et al 2013). Der flächige Fortbestand dieses Buchsbestandes wird als unwahrscheinlich eingestuft (John \& Schumacher 2013).

\section{Edelkastanien-Gallwespe}

Die aus Südchina stammende Kastanien-Gallwespe (Dryocosmus kuriphilus) lebt im Ursprungsgebiet auf verschiedenen Castanea-Arten. Sie wurde weltweit verschleppt und 2002 mit Pflanzenmaterial aus Japan auch nach Europa, ins Piemont, eingeführt. 2009 wurde sie im Tessin entdeckt (Forster et al 2009). Die nur rund drei Millimeter kleinen Wespen vermehren sich ausschliesslich ungeschlechtlich - interessanterweise konnten bisher keine Männchen nachgewiesen werden. Jedes Weibchen legt im Sommer bis etwa ein Dutzend Eier in eine Kastanienknospe ab. Insgesamt kann es rund 100 Eier produzieren. Nach einem Monat schlüpfen die kleinen Eilarven aus, verharren aber bis zum Austrieb im nächsten Frühling in einer Ruhephase in der Knospe. Beim Austrieb bewirkt der Frass der Larven eine schnelle Bildung mehrkammriger Gallen an Blättern, Trieben und selten auch an Blüten (Abbildung 4). Ab Juni fliegen die adulten Wespen aus und suchen zur Eiablage neue Knospen auf. Die Generation ist somit einjährig. Die verlassenen Gallen werden braun und vertrocknen, bleiben aber noch bis zwei Jahre am Baum sichtbar. Durch die Vergallung wird die Assimilationsfläche reduziert und die Produktion von Kastanienfrüchten beeinträchtigt (Kato \& Hijii 1997). Die verlassenen Gallen erleichtern möglicherweise auch dem Kastanienrindenkrebs die Besiedlung eines Baumes (Prospero \& Forster 2011).

Die Ausbreitungsleistung der Gallwespen beträgt im Allgemeinen einige Kilometer pro Jahr. Mittlerweile sind praktisch der ganze Kanton Tessin und die Bündner Südtäler betroffen. Mit Pflanzmaterial gelangte die Gallwespe auch an den Genfersee und hat sich dort ausgebreitet. Auch im Mittelland haben lokale Verschleppungen zu Befall von Einzelbäumen und Beständen von Edelkastanien geführt (Abbildung 5). Eine Untersuchung im Tessin zeigte, dass der Anteil von fehlentwickelten Gallenkammern in den ersten fünf Jahren von 7\% auf 39\% anstieg (Bloch 2012). Einheimische Schlupfwespen und Pathogene befallen zwar die Dryocosmus-Larven, waren bisher aber wenig erfolgreich. Im angrenzenden Italien wurde 2005 zur Bekämpfung von D. kuriphilus auch die aus Japan importierte Schlupfwespe 


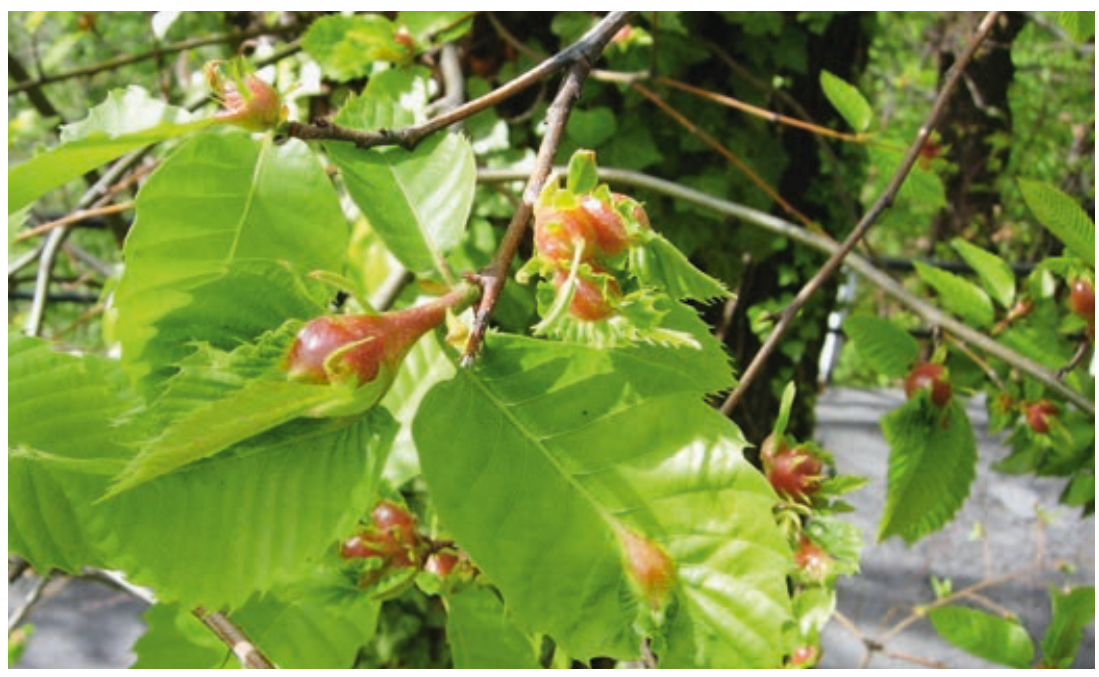

Abb 4 Blattgallen der Edelkastanien-Gallwespe (Dryocosmus kuriphilus) an verschiedenen Stellen der Blätter.

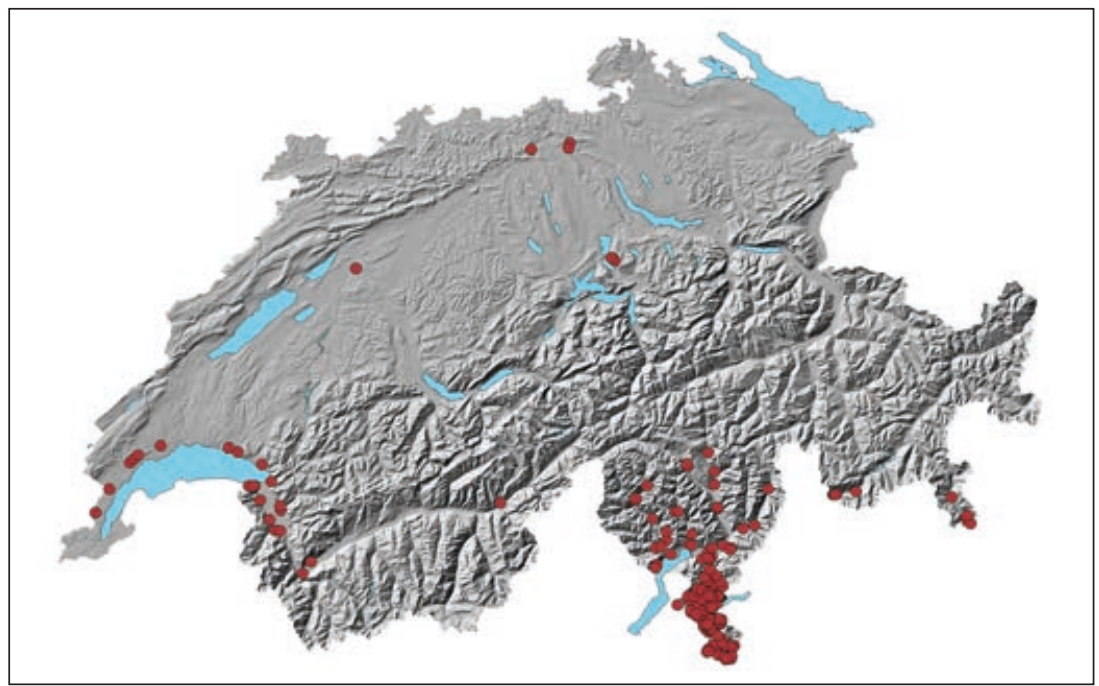

Abb 5 Verbreitung der Kastanien-Gallwespe (Dryocosmus kuriphilus) in der Schweiz, Stand Ende 2013. Quelle: Waldschutz Schweiz, WSL

Torymus sinensis freigesetzt. Diese wurde 2013 auch im Tessin festgestellt (Meier et al 2014).

Die Kastanien-Gallwespe gehört in Europa und in der Schweiz zu den «besonders gefährlichen Schadorganismen» (Quarantäneorganismen) und ist damit melde- und bekämpfungspflichtig. Allerdings ist der Befall im Tessin und in der Westschweiz so ausgedehnt, dass dort keine Bekämpfungsmassnahmen mehr durchgeführt werden können. Aus den Befallsgebieten darf jedoch kein Pflanzenmaterial von Edelkastanien in befallsfreie Gebiete ausgeführt werden, ausgenommen Holz und Früchte. Auf der Alpennordseite muss jeder neue Befall oder Verdachtsfall dem kantonalen Pflanzenschutzdienst gemeldet werden. Da befallene Knospen sich äusserlich nicht von unbefallenen unterscheiden, erfolgt die Kontrolle von Pflanzenmaterial am besten im Frühling nach dem Blattaustrieb, wenn die Gallen sichtbar werden.

\section{Asiatischer Laubholzbockkäfer}

In der Deutschschweiz hat unter den Neueinschleppungen der Asiatische Laubholzbockkäfer
(Anoplophora glabripennis) die grösste Bedeutung. Dieser ostasiatische Käfer wurde in den 1990er-Jahren in die USA und Anfang dieses Jahrzehnts nach Europa eingeschleppt. In der Schweiz fand man 2011 in Brünisried im Kanton Freiburg erstmals adulte Käfer wie auch frische Eiablagen an Ahornen. Ein Jahr später sorgte einer der grössten Befallsherde Europas in der Stadt Winterthur für grosses mediales Aufsehen. Die bis über drei Zentimeter grossen Käfer befallen gesunde Bäume fast aller Laubbaumarten, bevorzugt Ahorn (Acer), Weide (Salix), Birke (Betula), Pappel (Populus) und Rosskastanie. Sie legen ihre Eier in die Rinde $a b$, die ausgeschlüpften Larven fressen zuerst im Bast und dringen dann in den Holzkörper ein. Am Ende der zweijährigen Entwicklung verpuppen sich die rund fünf Zentimeter grossen Larven. Der fertige Käfer bohrt sich durch ein rund ein Zentimeter weites, rundes Loch aus dem Ast oder Stamm (Abbildung 6). Nach dem Reifungsfrass an grüner Rinde legen die Weibchen ihre Eier häufig in unmittelbarer Umgebung ab, falls geeignete Bäume vorhanden sind.

Jungbäume beginnen nach einem starken Befall schnell zu kränkeln und abzusterben, ausgewachsene Bäume ertragen ihn aber längere Zeit relativ gut. Der Käfer steht in ganz Europa unter Quarantäne, und ein Befall muss dem kantonalen Pflanzenschutzdienst gemeldet und sofort getilgt werden. Dies soll eine weitere Käferausbreitung verhindern und vermindert im Siedlungsraum das Risiko von Personenund Sachschäden infolge von Astbrüchen. Die in den USA bis 2008 angefallenen Kosten von 373 Millionen Dollar für Bekämpfungsmassnahmen, Monitoring und Neubepflanzungen (Haack et al 2010) zeigen, dass diese Massnahmen ins Geld gehen können.

Der Asiatische Laubholzbockkäfer wird vor allem mit Verpackungsholz verschleppt. Insbesondere aus China stammende Lattenkisten mit Granitsteinen enthielten in der Vergangenheit oft Larven dieser Käfer, die sich in Europa fertig entwickeln konnten und zu Befall in der Umgebung führten. So lag der Herd des Winterthurer Befalls an einer Strassenkreuzung, wo einige Jahre zuvor Paletten mit Granitsteinen aus China für den Strassenbau gelagert waren. Weitere Informationen sowie Verwechslungsmöglichkeiten mit einheimischen Arten wurden in einem Merkblatt zusammengestellt (Wermelinger et al 2013).

Da praktisch alle Laubbaumarten der Schweiz auf der Wirtsliste dieses äusserst polyphagen Bockkäfers stehen, stellt sich die Frage nach dem Befallsrisiko im Wald. In Europa sind bisher zwei Fälle aus Österreich und Deutschland bekannt, bei denen jeweils ein Wäldchen befallen wurde. Ein grösseres Ausmass wurde in Massachusetts in den USA erreicht, wo in Laubmischwäldern ausschliesslich Ahorne verschiedener Arten befallen wurden (Dodds \& Orwig 2011). Interessant ist, dass von den befallenen Bäumen bisher noch keiner abgestorben ist. Für den 


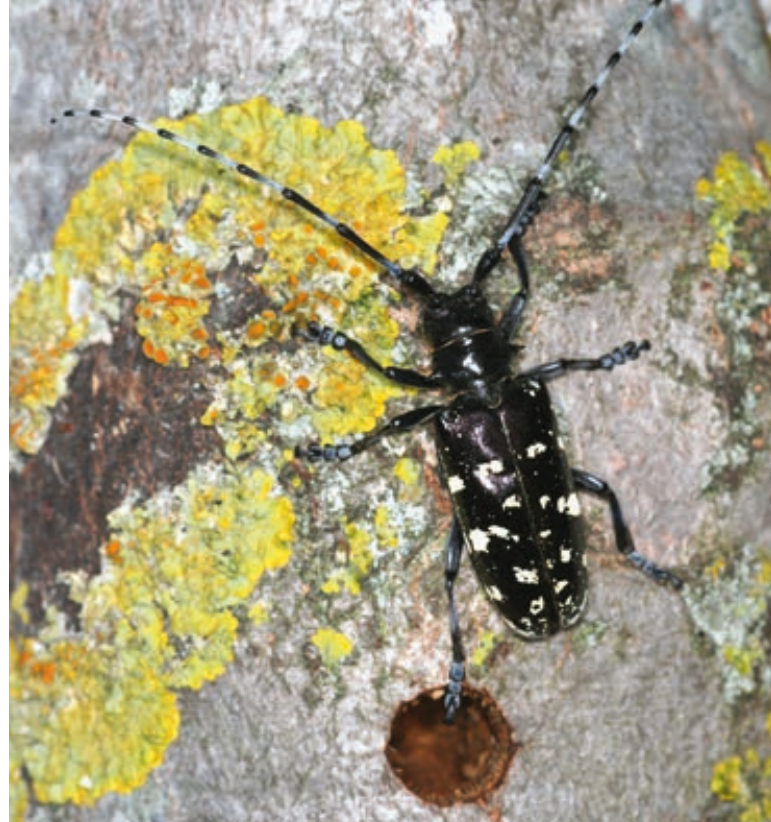

Abb 6 Der auffällige Asiatische Laubholzbockkäfer (Anoplophora glabripennis) bei einem Ausbohrloch an der bevorzugten Baumart Ahorn.

Schweizer Wald stellen sich konkret verschiedene Fragen: Wird sich der Asiatische Laubholzbockkäfer im Wald etablieren können? Welche Baumarten wird er besiedeln? Werden Bäume deswegen absterben (abgesehen von den Tilgungsmassnahmen)? Welche ökonomischen und ökologischen Konsequenzen hätte eine ausgedehnte Mortalität zum Beispiel von Ahorn? Im Moment kann darüber nur spekuliert werden. Der Übergang dieses Käfers in den Wald ist zwar sehr wohl möglich, ein grossflächiger Ausfall bestimmter Baumarten aber eher unwahrscheinlich. Im Gegensatz zu Strassen- und Parkbepflanzungen ist ein Wald ein besser gepuffertes System, wo natürliche Regulationsfaktoren wie beispielsweise Spechte - die wichtigsten Antagonisten im Ursprungsland China - besser greifen können.

\section{Welches sind die nächsten Kandidaten?}

Trotz vorgeschriebener chemischer oder thermischer Behandlung von Verpackungsholz und intensivierten Kontrollmassnahmen in den Exportund Importländern werden sich auch in Zukunft weitere neue und darunter auch invasive Insektenarten bei uns einnisten. Ein solcher Kandidat ist der Citrusbockkäfer (Anoplophora chinensis), ein naher Verwandter des Asiatischen Laubholzbockkäfers. Die beiden Arten sehen praktisch gleich aus, haben dieselbe Biologie und ein ähnlich breites Wirtsbaumspektrum, unterscheiden sich aber in zwei wesentlichen Aspekten: Der Citrusbockkäfer entwickelt sich im Gegensatz zum Asiatischen Laubholzbockkäfer mehrheitlich am Stammfuss und in den Wurzeln. Weiter erfolgt die Verschleppung des Citrusbocks nicht mit Verpackungsholz, sondern in lebenden
Zierpflanzen. Einzelne solcher Käfer und Larven wurden in der Schweiz bereits abgefangen, die Art konnte sich aber noch nicht vermehren. Im Raum Mailand hat sich jedoch ein über 400 Quadratkilometer grosser Befallsherd dieses Käfers gebildet. Es scheint nur eine Frage der Zeit, bis der Käfer mit Pflanzenmaterial oder selber fliegend das Tessin erreicht.

Ein weiterer Kandidat asiatischen Ursprungs ist der Palmrüssler (Rhynchophorus ferrugineus; Abbildung 7). Er wurde durch den Import von Palmen in den Mittelmeerraum eingeschleppt und breitet sich Richtung Norden aus. Er dürfte entweder mit Pflanzenmaterial oder aktiv fliegend von Italien her bald das Tessin erreichen. Er befällt sämtliche Palmenarten und bringt sie zum Absterben, da sich die Larven im Vegetationskegel der Pflanzen entwickeln. Ähnliches gilt für den Palmenschmetterling Paysandisia archon. Ob dies in Bezug auf die sich im Tessin auch in den Wald ausbreitenden Palmen negativ oder positiv zu werten ist, sei dahingestellt. Eine grössere Bedrohung im Schweizer Wald stellt der Eschenprachtkäfer (Agrilus planipennis) dar. Er befällt ausschliesslich Eschen, die in Europa bekanntlich bereits durch die Eschenwelke unter Druck stehen, und tötet sie innert kurzer Zeit ab. Diese ebenfalls ostasiatische Art wurde vor rund zehn Jahren in Nordamerika eingeschleppt und hat dort als der berüchtigte «Emerald Ash Borer» bereits den Tod von Millionen von Eschen im urbanen Grünbereich und im Wald verursacht. Seit 2003 ist der ausbreitungsstarke Käfer auch an Bäumen in der Grossregion Moskau zu finden und wird schon bald die Grenze zu Weissrussland erreichen (Orlova-Bienkowskaja 2013). Die Art wird sich wohl über kurz oder lang bei uns einfinden.

\section{Einfluss des Klimawandels}

Wie bereits erwähnt, kann eine Folge der höheren Temperaturen sein, dass mehr mediterrane Arten in nördlichere Gebiete einwandern. Auf die Verschleppung von Arten aus anderen Kontinenten hat der Klimawandel direkt keinen Einfluss. Wohl aber können die veränderten Bedingungen am Zielort das Etablieren und die Vermehrung einer neuen Art erleichtern. Insbesondere können sich mildere Winter positiv auf die Überlebensraten exotischer Arten auswirken und eine Entwicklung an Orten ermöglichen, die vorher zu kalt waren (Walther et al 2009). Auch die Physiologie der Wirtspflanzen, exotischen wie einheimischen, könnte sich infolge des globalen Wandels verändern und die Pflanzen für Neozoen attraktiver machen. Ein höherer $\mathrm{CO}_{2}$-Gehalt der Luft, vermehrte Deposition von Stickstoff und Luftschadstoffe können die Zusammensetzung der Pflanzen zugunsten von herbivoren Insekten verändern (Dukes \& Mooney 1999). Speziell die prognostizierte zunehmende Sommertrockenheit dürfte die Widerstands- 


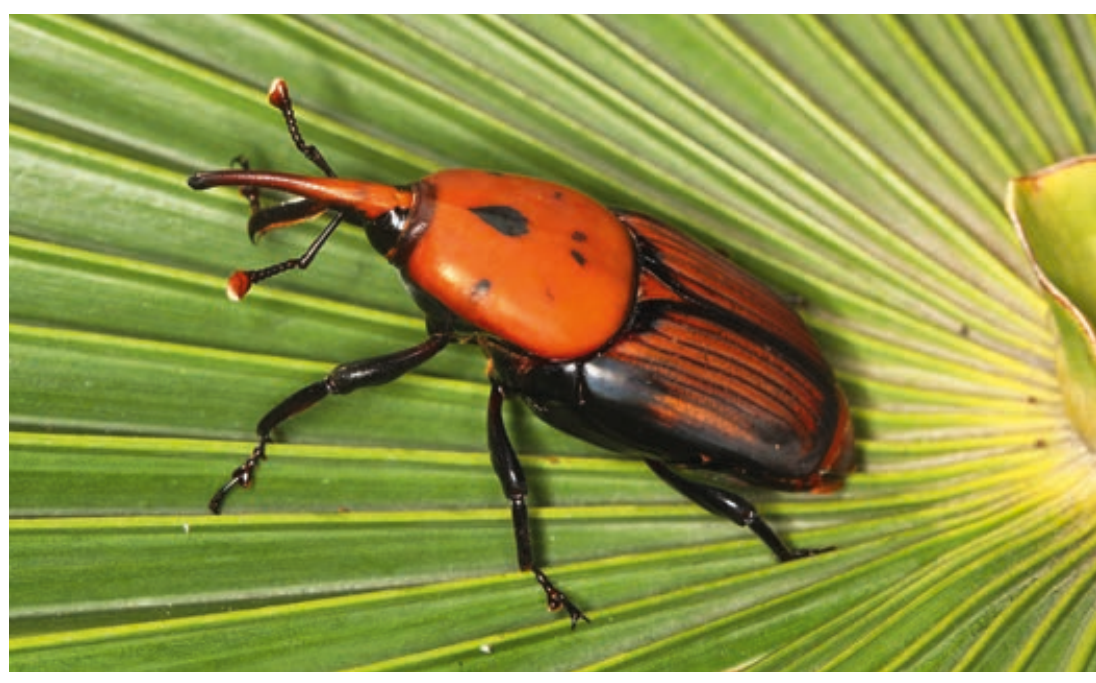

Abb 7 Der Palmrüssler (Rhynchophorus ferrugineus): schön, aber für Palmen tödlich.

kraft der Wirtspflanzen gegen Befall durch Neozoen (und heimische Schädlinge) herabsetzen. Infolge der oben erwähnten Einflüsse und eines sich ändernden Störungsregimes können eingeschleppte Arten auch von neuen ökologischen Nischen profitieren.

\section{Handlungsoptionen}

Im Management von Neozoen gilt es in erster Linie zu verhindern, dass invasive Arten überhaupt zu uns gelangen. Das Beispiel des Asiatischen Marienkäfers zeigt, dass der Einsatz fremdländischer «Nützlinge» sehr gewissenhafter Abklärung der Nebenwirkungen auf andere Organismen bedarf. Kontinentfremde Arten stossen auf Ökosysteme, mit denen sie nicht koevoluiert haben und die deshalb empfindlich auf neu eingeführte Komponenten reagieren (Waring \& O'Hara 2005).

Die phytosanitäre Kontrolle von Handelsware konzentriert sich aus Kapazitätsgründen weltweit vor allem auf bekannte und aktuelle Probleminsekten, was das Einschleppen von anderen Arten nur teilweise eindämmt. Während in Nordamerika die Einschleppungsraten seit einem halben Jahrhundert rückläufig sind, steigen sie in Europa immer noch steil an (Roques 2010b). Dies zeigt, dass die Kontrollen an Europas Grenzen ungenügend sind. Innerhalb des EU-Raums gibt es kaum mehr Zollkontrollen, was die Ausbreitung von einmal eingeschleppten Arten vereinfacht. Importe von lebenden Bäumen, Sträuchern und Bonsais sind der grösste Risikofaktor (Roques 2010b) und sollten stärker eingeschränkt und noch besser kontrolliert werden. Alle lebenden Pflanzen aus Drittländern (Nicht-EU-Staaten) müssen zwar heute von einem Pflanzenschutzzeugnis begleitet sein und werden stichprobenweise durch destruktives Zerlegen kontrolliert. Dennoch bleibt die Kontrolle lebender Pflanzen in Europa ein Schwachpunkt.

Verpackungsholz von Steinprodukten aus Drittstaaten muss nach internationalem Standard
(ISPM 15) thermisch oder chemisch behandelt sein, was vom Eidgenössischen Pflanzenschutzdienst seit 2012 intensiv kontrolliert wird. Bei Beanstandungen muss das Verpackungsholz umgehend vernichtet werden. Es ist auch zu prüfen, inwieweit das Verpackungsholz ganz weggelassen oder durch andere Materialien ersetzt werden kann.

Haben Neozoen mit invasivem Charakter bereits erste Befallsherde bilden können, wird die Strategie der Tilgung verfolgt. Auch dies wird vom Eidgenössischen Pflanzenschutzdienst veranlasst, und die kantonalen Dienste sind für die Durchführung verantwortlich. Dabei gilt es, den gesamten neu auftretenden Befall zu eliminieren, bis ein Gebiet wieder als befallsfrei betrachtet werden kann. Ist ein Befall wie bei der Edelkastanien-Gallwespe so weit fortgeschritten, dass eine Tilgung aussichtslos geworden ist, kann nur versucht werden, die Ausbreitung des Organismus einzudämmen. Die Bekämpfung erfolgt dann nach den gleichen Grundsätzen wie bei heimischen Schadorganismen.

Da ein Befall meistens zuerst an Zierpflanzen auftritt, ist eine entsprechende Ausbildung des Personals in Gärtnereien, Baumschulen und im städtischen Grün äusserst wichtig. Diese Betriebe stellen eine Art Frühwarnsystem für den Wald dar. Deshalb muss der Informationsaustausch bezüglich neuer Krankheiten und Schädlinge von Sträuchern und Bäumen zwischen urbanem Grün, Land- und Waldwirtschaft, Baumpflege sowie Behörden verbessert und systematisiert werden.

Vor dem Hintergrund des Klimawandels wird vermehrt der Einsatz von Gastbaumarten im Schweizer Wald diskutiert. Dabei müssen auch die Risiken durch invasive Insekten (und Krankheiten) berücksichtigt werden. Exotische Baumarten ermöglichen eingeschleppten, spezialisierten Neozoen erst ihre Entwicklung. Die Bäume befinden sich hier in einem fremden Umfeld, das wahrscheinlich nicht die adäquaten regulatorischen Fähigkeiten bezüglich dieser Insekten hat wie das Herkunftsgebiet. Wie für einheimische Schadinsekten sind auch für Neozoen waldbauliche Strategien nötig, die auf eine standortgerechte und vielfältige Mischung möglichst einheimischer Baumarten mit hoher Vitalität und Resistenz setzen.

Eingereicht: 31. Oktober 2013, akzeptiert (mit Review): 23. Januar 2014

\section{Dank}

Ich bedanke mich bei Waldschutz Schweiz (WSL) für die Abbildung 5 und für das sorgfältige Gegenlesen des Manuskripts sowie bei Ernst Fürst (BAFU) für Kommentare zu einzelnen Abschnitten. Ebenso danke ich Silvia Dingwall für die Durchsicht des englischen Abstracts und Monique Dousse für die französische Übersetzung des Abstracts. 


\section{Literatur}

BATTISTI A, STASTNY M, NETHERER S, ROBINET C, SCHOPF A (2005) Expansion of geographic range in the pine processionary moth caused by increased winter temperatures. Ecol Appl 15: 2084-2096.

BLOCH ME (2012) Chestnut gall wasp (Dryocosmus kuriphilus Yasum.) on sweet chestnut (Castanea sativa): Gall characteristics and early epidemic evolution under natural conditions. Zürich: Eidg Techn Hochschule, Umweltsystemwissenschaften, Master Thesis. 57 p.

DODDS KJ, ORWIG DA (2011) An invasive urban forest pest invades natural environments - Asian longhorned beetle in northeastern US hardwood forests. Can J For Res 41: 1729-1742.

DUKES JS, MOONEY HA (1999) Does global change increase the success of biological invaders? Trends Ecol Evol 14: 135-139.

FORSTER B ET AL (2009) Die Edelkastaniengallwespe Dryocosmus kuriphilus (Yasumatsu) (Hymenoptera, Cynipidae) tritt erstmals in der Südschweiz auf. Mitt Schweiz Entomol Ges 82: 271-279.

HAACK RA, HERARD F, SUN JH, TURGEON JJ (2010) Managing invasive populations of Asian Longhorned Beetle and Citrus Longhorned Beetle: a worldwide perspective. Annu Rev Entomol 55: 521-546.

JOHN R, SCHUMACHER J (2013) Der Buchsbaum-Zünsler (Cydalima perspectalis) im Grenzach-Wyhlener Buchswald - Invasionschronik und Monitoringergebnisse. Gesunde Pflanzen 65: 1-6.

KATO K, HIJII N (1997) Effects of gall formation by Dryocosmus kuriphilus Yasumatsu (Hym., Cynipidae) on the growth of chestnut trees. J Appl Entomol 121: 9-15.

KENIS M (2006) Insekten - Insecta. In: Wittenberg R, editor. Gebietsfremde Arten in der Schweiz. Eine Übersicht über gebietsfremde Arten und ihre Bedrohung für die biologische Vielfalt und die Wirtschaft der Schweiz. Bern: Bundesamt Umwelt. pp. 66-97.

KENIS M, BRANCO M (2010) Impact of alien terrestrial arthropods in Europe, Chapter 5. BioRisk 4: 51-71.

\section{Invasive Gehölzinsekten: Bedrohung für den Schweizer Wald?}

Die Rate der Einschleppung gebietsfremder Insekten und anderer Gliederfüsser nach Europa ist in den letzten Jahrzehnten exponentiell angestiegen. Im vergangenen Jahrzehnt betrug sie im Schnitt 20 Arten pro Jahr, seit der Entdeckung Amerikas bis zum Jahr 2008 waren es insgesamt 1590 neue Arten. Hauptgrund dafür ist der intensivierte und globalisierte Warenhandel. Die wichtigsten Einschleppungswege sind der Zierpflanzenhandel und der Import von Waren in befallenem Verpackungsholz. Neue Arten etablieren sich meist zuerst im Siedlungsbereich, wo das vergleichsweise milde Klima das Überleben exotischer Arten begünstigt. Im Artikel werden drei kürzlich eingeschleppte, waldrelevante Arten vorgestellt, die alle aus dem ostasiatischen Raum stammen. Der Buchsbaumzünsler (Cydalima perspectalis) befiel im Raum Basel natürliche Buchsbestände im Wald. Die EdelkastanienGallwespe (Dryocosmus kuriphilus) ist auf der Alpensüdseite praktisch flächendeckend vorhanden und weist auch im Mittelland einzelne Befallsherde auf. Vom Asiatischen Laubholzbockkäfer (Anoplophora glabripennis) sind in der Schweiz zwei aktive Befallsherde bekannt. Zudem werden mögliche Kandidaten weiterer invasiver Insekten diskutiert. Ein grossflächiges Einbringen von Gastbaumarten im Wald als Wirtspflanzen jetziger und künftiger invasiver Insekten ist kritisch zu beurteilen.
KENIS M, NACAMBO S, LEUTHARDT FLG, DI DOMENICO F, HAYE T (2013) The box tree moth, Cydalima perspectalis, in Europe: horticultural pest or environmental disaster? Aliens 33: 38-41.

MEIER F, ENGESSER R, FORSTER B, ODERMATT O, ANGST A (2013) Forstschutz-Überblick 2012. Birmensdorf: Eidgenöss Forsch. anstalt WSL. $30 \mathrm{p}$.

MEIER F, ENGESSER R, FORSTER B, ODERMATT O, ANGST A (2014) Forstschutz-Überblick 2013. Birmensdorf: Eidgenöss Forsch. anstalt WSL. 28 p.

ORLOVA-BIENKOWSKAJA MJ (2013) Ashes in Europe are in danger: the invasive range of Agrilus planipennis in European Russia is expanding. Biol Invas, doi: 10.1007/s10530-013-0579-8.

PROSPERO S, FORSTER B (2011) Chestnut gall wasp (Dryocosmus kuriphilus) infestations: new opportunities for the chestnut blight fungus Cryphonectria parasitica? New Disease Reports 23: 35 .

ROQUES A (2010A) Taxonomy, time and geographic patterns Chapter 2. BioRisk 4: 11-26.

ROQUES A (2010B) Alien forest insects in a warmer world and a globalised economy: impacts of changes in trade, tourism and climate on forest biosecurity. NZ J For Sci 40: S77-S94.

ROY HE ET AL (2012) Invasive alien predator causes rapid declines of native European ladybirds. Diversity Distrib 18: 717-725.

SMITH RM ET AL (2007) Recent non-native invertebrate plant pest establishments in Great Britain: origins, pathways, and trends. Agric For Entomol 9: 307-326.

WALTHER GR ET AL (2009) Alien species in a warmer world: risks and opportunities. Trends Ecol Evol 24: 686-693.

WARING KM, O'HARA KL (2005) Silvicultural strategies in forest ecosystems affected by introduced pests. For Ecol Manage 209: 27-41.

WERMELINGER B ET AL (2013) Invasive Laubholz-Bockkäfer aus Asien - Ökologie und Management. Birmensdorf: Eidgenöss Forsch.anstalt WSL, Merkbl Prax 50. 16 p.

WESTPHAL MI, BROWNE M, MACKINNON K, NOBLE I (2008) The link between international trade and the global distribution of invasive alien species. Biol Invasions 10: 391-398.

\section{Les insectes envahissants des arbres: une menace pour la forêt suisse?}

La quantité d'insectes exotiques et d'autres arthropodes introduits en Europe a augmenté de manière exponentielle au cours des décennies passées. Elle se chiffre à 20 espèces par an en moyenne pour la dernière décennie, alors que depuis la découverte de l'Amérique jusqu'en 2008, un total de 1590 nouvelles espèces $s^{\prime} y$ étaient introduites. L'intensification et la globalisation du commerce des marchandises en sont la cause majeure. Les principales voies d'introduction sont le commerce de plantes ornementales et l'importation de marchandises se trouvant dans du bois d'emballage infesté. Les nouvelles espèces s'installent souvent d'abord dans les zones urbanisées. Le climat plus doux de ces zones favorise généralement la survie des espèces exotiques. Trois espèces de I'Asie de l'Est récemment introduites en Suisse et pouvant être nuisibles en forêt sont présentées dans cet article: La pyrale du buis (Cydalima perspectalis) sévit en forêt dans des peuplements naturels de buis de la région bâloise. Le cynips du châtaignier (Dryocosmus kuriphilus) est répandu sur presque tout le versant sud des Alpes et dans la région lémanique ainsi que ponctuellement sur le Plateau. Pour le capricorne asiatique (Anoplophora glabripennis), dont les larves peuvent se développer dans la plupart des essences feuillues, deux foyers d'infestation sont connus en Suisse. Une introduction à large échelle d'espèces ligneuses exotiques en forêt mérite un examen critique en considérant ces arbres comme autant de plantes-hôtes des actuels et futurs insectes envahissants. 